TRANSACTIONS OF THE

AMERICAN MATHEMATICAL SOCIETY

Volume 355, Number 1, Pages 265-271

S 0002-9947(02)03108-2

Article electronically published on September 5, 2002

\title{
ON GRAPHIC BERNSTEIN TYPE RESULTS IN HIGHER CODIMENSION
}

\author{
MU-TAO WANG
}

\begin{abstract}
Let $\Sigma$ be a minimal submanifold of $\mathbb{R}^{n+m}$ that can be represented as the graph of a smooth map $f: \mathbb{R}^{n} \mapsto \mathbb{R}^{m}$. We apply a formula that we derived in the study of mean curvature flow to obtain conditions under which $\Sigma$ must be an affine subspace. Our result covers all known ones in the general case. The conditions are stated in terms of the singular values of $d f$.
\end{abstract}

\section{INTRODUCTION}

The well-known Bernstein theorem asserts that any complete minimal surface that can be written as the graph of a function on $\mathbb{R}^{2}$ must be a plane. This result has been generalized to $\mathbb{R}^{n}$, for $n \leq 7$ and to general dimensions under various growth conditions; see [2] and the references therein for the codimension one case. In this note, we study the higher codimension case, i.e., minimal submanifolds in $\mathbb{R}^{n+m}$ that can be written as graphs of vector-valued functions $f: \mathbb{R}^{n} \mapsto \mathbb{R}^{m}$.

For higher codimension Bernstein type problems, there are general results of [3], [5] and [7. The idea in these papers is to find a subharmonic function whose vanishing implies that $\Sigma$ is totally geodesic. Under the assumption that $f$ is of bounded gradient, one can perform blow-down analysis to reduce to the minimal cone case. The maximum principle together with the Allard regularity theorem then complete the argument. A fundamental fact is that the Gauss map of a minimal submanifold is a harmonic map; so any convex function on the Grassmannian renders a subharmonic function. The work in [3], [5], and 7] consists of delicate analysis of the geometry of the Grassmannian in order to locate the maximal region where a convex function exists. The condition for Bernstein type results is in terms of the function

$$
* \Omega_{1}=\frac{1}{\sqrt{\operatorname{det}\left(I+(d f)^{t} d f\right)}} .
$$

This can be considered as the inner product of the tangent space of $\Sigma$ and the domain $\mathbb{R}^{n}$. In particular, in [3] and [5], it was shown that

$$
* \Omega_{1} \geq K^{\prime}>\cos ^{p}\left(\frac{\pi}{2 \sqrt{2} p}\right)
$$

where $p=\min (n, m)$ implies that $\Sigma$ is an affine subspace. This bound is later improved in [7] to

$$
* \Omega_{1} \geq K^{\prime \prime}>\frac{1}{2} .
$$

Received by the editors February 6, 2002 .

2000 Mathematics Subject Classification. Primary 53A10, 35J50, 53A07, 49Q05, 53C38.

The author was supported by NSF grant DMS 0104163. 
We adopt a direct approach to calculate explicitly the Laplacian of $\ln * \Omega$. The calculation first appeared to us in [13] in the context of mean curvature flow. The formula determines precisely when $\ln * \Omega$ is a superharmonic function.

Theorem 1.1. Let $\Sigma$ be a minimal submanifold of $\mathbb{R}^{n+m}$. Suppose $\Sigma$ can be written as the graph of a smooth map $f: \mathbb{R}^{n} \mapsto \mathbb{R}^{m}$. Denote the singular values of $d f$ by $\lambda_{i}, i=1, \cdots, n$. If there exist $\delta, K>0$ such that $\left|\lambda_{i} \lambda_{j}\right| \leq 1-\delta$ for any $i \neq j$ and $* \Omega_{1}=\frac{1}{\sqrt{\operatorname{det}\left(I+(d f)^{t} d f\right)}} \geq K$, then $\Sigma$ is an affine subspace.

The condition $\left|\lambda_{i} \lambda_{j}\right|<1$ means $d f$ is area-decreasing on any two-dimensional subspaces. Notice that $K$ is not necessarily bounded from below. At the end of $\S 3$, we discuss how Theorem A implies the results in [3], [5] or [7].

We remark that Theorem A also holds for submanifolds with parallel mean curvature vector. This condition for minimal submanifolds is not the optimal one. We state it first because it is simple to apply and seems natural (see the remark at the end of the introduction on the geometric meaning). The optimal condition, which is in terms of $\lambda_{i}$ again, is somewhat more complicated. It will be described explicitly in $\S 2$.

Theorem 1.2. Under the assumption of Theorem A, there exists an optimal condition in terms of the singular values of df such that $\Sigma$ is an affine subspace whenever $f$ satisfies this optimal condition.

Theorem B also implies the result of 1] and [3] that any three-dimensional minimal cone is flat and thus is sharper than Theorem A.

The higher codimension Bernstein type result is not expected to be true in the most generality due to the counterexample of Lawson and Osserman 9].

Notice that in the codimension one case, any graphic minimal hypersurface is stable. It is thus natural to impose the stable assumption in higher codimension. A special category of minimal submanifolds is calibrated submanifolds, studied by Harvey and Lawson [6]. They are volume minimizing and thus stable. These include holomorphic submanifolds, special Lagrangian submanifolds, associative, co-associative, and Cayley submanifolds. The special Lagrangian case is studied in [4, 10], 8], 14], and [11. The Lagrangian condition implies the existence of a potential function $F$ such that $f=\nabla F$. In terms of PDE, the Bernstein type problem asks when an entire solution of

$$
\operatorname{Im}\left(\operatorname{det}\left(I+\sqrt{-1} D^{2} F\right)\right)=0
$$

is a quadratic polynomial. In this case, the condition of Theorem A or Theorem B can be stated in terms of the eigenvalues of $D^{2} F$.

We remark that conditions on the singular values of $d f$ or eigenvalues of $D^{2} F$ depend on the choice of a subspace (usually a calibrated one) over which $\Sigma$ is written as a graph. Such conditions correspond to regions in the relevant Grassmannian. A more invariant description is to require that the image of the Gauss map lie in the orbit of one of these regions under the respective holonomy group actions.

To demonstrate, let us compare the conditions $\left|\lambda_{1} \lambda_{2}\right|<1$ and $\left(1+\lambda_{1}^{2}\right)\left(1+\lambda_{2}^{2}\right)<4$ (or $* \Omega_{1}=\frac{1}{\sqrt{\left(1+\lambda_{1}^{2}\right)\left(1+\lambda_{2}^{2}\right)}}>\frac{1}{2}$ ) when $n=m=2$. It is clear that the former condition is weaker than the latter one. In this case, $G(2,4)=S^{2}\left(\frac{1}{\sqrt{2}}\right) \times S^{2}\left(\frac{1}{\sqrt{2}}\right)$. In $f: \mathbb{R}^{2} \mapsto \mathbb{R}^{2}$, denote the coordinates on the domain $\mathbb{R}^{2}$ by $x^{1}, x^{2}$ and the 
coordinates on the target $\mathbb{R}^{2}$ by $y^{1}, y^{2}$. Then the forms $\omega_{1}, \omega_{2}$ given by

$$
\begin{aligned}
& \omega_{1}=\frac{1}{\sqrt{2}}\left(d x^{1} \wedge d x^{2}-d y^{1} \wedge d y^{2}\right), \\
& \omega_{2}=\frac{1}{\sqrt{2}}\left(d x^{1} \wedge d x^{2}+d y^{1} \wedge d y^{2}\right)
\end{aligned}
$$

serve as coordinate functions on $G(2,4)$; see, for example, section 3 in 12 . We may consider them as the height functions on each of the two $S^{2}\left(\frac{1}{\sqrt{2}}\right)$. The condition $\left|\lambda_{1} \lambda_{2}\right|<1$ corresponds to $* \omega_{1}>0$ and $* \omega_{2}>0$, which means that the image of the Gauss map lies in the product of the two hemispheres. These are natural geometric conditions. It is shown in [12] that, under this assumption, the maximum principle not only works in the elliptic case but also in the parabolic case.

Contrary to the codimension one case, the equation of the second fundamental form has not been used in higher codimension to our knowledge. We expect that a complete classification theorem of graphic calibrated submanifolds will rely on such an estimate.

The author wishes to thank Professor D. H. Phong and Professor S.-T. Yau for their encouragement and support. He also thanks Mao-Pei Tsui for useful discussions.

\section{General Results}

We first recall a formula whose parabolic version was derived in [13]. To apply the formula in [13] to the current situation, we note that a minimal submanifold corresponds to the stationary phase of mean curvature flow.

Let $\Sigma$ be an $n$-dimensional submanifold of $\mathbb{R}^{n+m}$ and $\Omega$ a parallel $n$-form on $\mathbb{R}^{n+m}$. Around any point $p \in \Sigma$, we choose any orthonormal frames $\left\{e_{i}\right\}_{i=1, \cdots, n}$ for $T_{p} \Sigma$ and $\left\{e_{\alpha}\right\}_{\alpha=n+1, \cdots, n+m}$ for $N_{p} \Sigma$, the normal bundle of $\Sigma$. The convention that $i, j, k, \cdots$ denote tangent indexes and $\alpha, \beta, \gamma \cdots$ denote normal indexes is followed.

The second fundamental form of $\Sigma$ is denoted by $h_{\alpha i j}=\left\langle\nabla_{e_{i}} e_{j}, e_{\alpha}\right\rangle$.

If we assume $\Sigma$ has parallel mean curvature vector, then $* \Omega=\Omega\left(e_{1}, \cdots, e_{n}\right)$ satisfies (see Proposition 3.1 in [13])

$$
\begin{aligned}
& \Delta * \Omega+* \Omega\left(\sum_{\alpha, l, k} h_{\alpha l k}^{2}\right)-2 \sum_{\alpha, \beta, k}\left[\Omega_{\alpha \beta 3 \cdots n} h_{\alpha 1 k} h_{\beta 2 k}+\Omega_{\alpha 2 \beta \cdots, n} h_{\alpha 1 k} h_{\beta 3 k}+\cdots\right. \\
& \left.+\Omega_{1 \cdots(n-2) \alpha \beta} h_{\alpha(n-1) k} h_{\beta n k}\right]=0 \\
& (* \Omega)_{k}=\sum_{\alpha} \Omega_{\alpha 2 \cdots n} h_{\alpha 1 k}+\cdots+\Omega_{1 \cdots n-1 \alpha} h_{\alpha n k}
\end{aligned}
$$

where $\Delta$ is the Laplace operator of the induced metric on $\Sigma$ and $\Omega_{\alpha \beta 3 \cdots n}=$ $\Omega\left(e_{\alpha}, e_{\beta}, e_{3}, \cdots, e_{n}\right)$.

Now take $\Omega=d x^{1} \wedge \cdots \wedge d x^{n}$, i.e., the volume form of $\mathbb{R}^{n}$. In this case, $* \Omega=$ $\Omega\left(e_{1}, \cdots, e_{n}\right)$ is exactly the Jacobian of the projection from $\Sigma$ to $\mathbb{R}^{n}$. A similar formula in this case appeared in Fischer-Colbrie's paper [3] (Lemma 1.1). 
When $m=1$, i.e., the codimension one case, $\partial_{i}=\frac{\partial}{\partial x^{i}}+\frac{\partial f}{\partial x^{i}} \frac{\partial}{\partial x^{n+1}}$ forms a basis for $T_{p} \Sigma$. Then

$$
* \Omega=\frac{\Omega\left(\partial_{1}, \cdots, \partial_{n}\right)}{\sqrt{\operatorname{det}\left(\partial_{i}, \partial_{j}\right)}} .
$$

It is not hard to compute that $* \Omega=\frac{1}{\sqrt{1+|d f|^{2}}}$, i.e., the angle made by the normal vector of $\Sigma$ and the $x_{n+1}$-axis. Thus formula (2.1) reduces to the classical formula for nonparametric minimal surfaces:

$$
\Delta \frac{1}{\sqrt{1+|d f|^{2}}}+\frac{1}{\sqrt{1+|d f|^{2}}}|A|^{2}=0
$$

A standard argument will imply the Bernstein type result for bounded $|d f|$.

In the general-dimensional case, we choose a particular basis to represent formula (2.1) for $\Omega=d x^{1} \wedge \cdots \wedge d x^{n}$. Given any $p$, then the differential of $f$ is a linear map from $\mathbb{R}^{n}$ to $\mathbb{R}^{m}$. We can use singular value decomposition to find orthonormal bases $\left\{a_{i}\right\}_{i=1, \cdots, n}$ for $\mathbb{R}^{n}$ and $\left\{a_{\alpha}\right\}_{\alpha=n+1, \cdots, n+m}$ for $\mathbb{R}^{m}$ such that

$$
d f\left(a_{i}\right)=\lambda_{i} a_{n+i}
$$

for $i=1, \cdots, n$. Notice that $\lambda_{i}=0$ if $i>\min \{n, m\}$.

Now

$$
\left\{e_{i}=\frac{1}{\sqrt{1+\lambda_{i}^{2}}}\left(a_{i}+\lambda_{i} a_{n+i}\right)\right\}_{i=1, \cdots, n}
$$

forms an orthonormal basis for $T_{p} \Sigma$ and

$$
\left\{e_{\alpha}=\frac{1}{\sqrt{1+\lambda_{\alpha-n}^{2}}}\left(a_{\alpha}-\lambda_{\alpha-n} a_{\alpha-n}\right)\right\}_{\alpha=n+1, \cdots, n+m}
$$

an orthonormal basis for $N_{p} \Sigma$.

It is not hard to see that $* \Omega=\frac{1}{\sqrt{\prod_{i=1}^{n}\left(1+\lambda_{i}^{2}\right)}}$. Applying these bases to equation (2.1), we obtain

Proposition 2.1. Let $\Sigma$ be the graph of a smooth map $f: \mathbb{R}^{n} \mapsto \mathbb{R}^{m}$ and let $\left\{\lambda_{i}\right\}_{i=1, \cdots, n}$ be the singular values of $d f$. If the mean curvature vector of $\Sigma$ is parallel, then $* \Omega$ satisfies the following equation:

$$
\Delta * \Omega=-* \Omega\left\{\sum_{\alpha, l, k} h_{\alpha l k}^{2}-2 \sum_{k, i<j} \lambda_{i} \lambda_{j} h_{n+i, i k} h_{n+j, j k}+2 \sum_{k, i<j} \lambda_{i} \lambda_{j} h_{n+j, i k} h_{n+i, j k}\right\}
$$

where $\Delta$ is the Laplace operator of the induced metric on $\Sigma$. The indexes $i, j$ range between 1 and $\min \{n, m\}$.

Proof of Theorem A. We shall calculate

$$
\Delta(\ln * \Omega)=\frac{* \Omega \Delta(* \Omega)-|\nabla * \Omega|^{2}}{|* \Omega|^{2}} .
$$

By formula (2.2), the covariant derivative of $* \Omega$ is

$$
(* \Omega)_{k}=-* \Omega\left(\sum_{i} \lambda_{i} h_{n+i, i k}\right) .
$$


Plug this and equation (2.1) into equation (2.4) and obtain

$$
\Delta(\ln * \Omega)=-\left\{\sum_{\alpha, l, k} h_{\alpha l k}^{2}+\sum_{k, i} \lambda_{i}^{2} h_{n+i, i k}^{2}+2 \sum_{k, i<j} \lambda_{i} \lambda_{j} h_{n+i, j k} h_{n+j, i k}\right\} .
$$

From the assumption of the theorem, it is obvious that $\Delta(\ln * \Omega) \leq-\delta_{1}|A|^{2}$ by completing the square. The condition $\frac{1}{\sqrt{\operatorname{det}\left(I+(d f)^{t} d f\right)}} \geq K$ means that $\Sigma$ is the graph of a vector-valued function with bounded gradient. Therefore, we can perform blow-down and dimension reduction to get a minimal cone. Notice that these conditions are invariant under scaling. We can apply the maximum principle to conclude that the minimal cone is flat, and then the Allard regularity theorem forces $\Sigma$ to be an affine space.

Proof of Theorem B. The optimal condition can be found by considering

$$
F\left(h_{\alpha l k}\right)=\sum_{\alpha, l, k} h_{\alpha l k}^{2}+\sum_{k, i} \lambda_{i}^{2} h_{n+i, i k}^{2}+2 \sum_{k, i<j} \lambda_{i} \lambda_{j} h_{n+i, j k} h_{n+j, i k}
$$

as a quadratic form defined on the space of all possible $h_{\alpha l k}$ with $h_{\alpha l k}=h_{\alpha k l}$ and $\sum_{k} h_{\alpha k k}=0$ for each $\alpha$. Now the problem is reduced to determining conditions on $\lambda_{i} \lambda_{j}$ to guarantee that $F$ is a positive quadratic form with $F\left(h_{\alpha l k}\right) \geq \epsilon \sum h_{\alpha l k}^{2}$ for some $\epsilon>0$. It is computable, yet it seems hard to find a simple description. At the end of the next section, we show how this gives the optimal result when $n=2$.

The condition in Theorem A or Theorem B depends on the choice of a subspace over which $\Sigma$ is written a graph. Since being minimal is invariant under the isometry action of $O(n+m)$, a slightly more general condition is the following.

Corollary 2.1. Let $\Sigma$ be the graph of $f: \mathbb{R}^{n} \mapsto \mathbb{R}^{m}$. Denote $A=d f$ as an $n \times m$ matrix. If there exists an element $g \in O(n+m)$ of the block form $g=\left[\begin{array}{ll}P & Q \\ R & S\end{array}\right]$ such that $P+A R$ is invertible and the singular values of $(P+A R)^{-1}(Q+A S)$ satisfy the optimal condition in Theorem $B$, then $\Sigma$ is an affine subspace.

Proof. The tangent space of $\Sigma$ is represented by $\left[\begin{array}{ll}I & A\end{array}\right]$. Under the action of $g$ on $\mathbb{R}^{n+m}$, the tangent space of $g(\Sigma)$ becomes $\left[\begin{array}{lll}P+A R & Q+A S\end{array}\right]$. If $P+A R$ is invertible, then $g(\Sigma)$ is still the graph of some $\bar{f}$ over some $\mathbb{R}^{n}$. Now $d \bar{f}=$ $(P+A R)^{-1}(Q+A S)$.

When $\Sigma$ is a Lagrangian submanifold defined as the graph of $\nabla F: \mathbb{R}^{n} \mapsto \mathbb{R}^{n}$, let $A=D^{2} F$. Note that being minimal Lagrangian is invariant under $U(n)$. An element $g$ in $U(n)$ can be expressed as a $2 n \times 2 n$ block matrix of the form

$$
\left[\begin{array}{cc}
P & -Q \\
Q & P
\end{array}\right]
$$

with

$$
P P^{t}+Q Q^{t}=I, \quad-P Q^{t}+Q P^{t}=0
$$

where the first $n$ components correspond to the real $x^{i}$ and the last $n$ components the imaginary $y^{i}$.

Rotating $\mathbb{C}^{n}$ by this element $g$, the tangent space becomes $\left[\begin{array}{ll}P+A Q & -Q+A P\end{array}\right]$. If we require $P+A Q$ to be invertible, then the condition is determined by the 
eigenvalues of the symmetric matrix $(P+A Q)^{-1}(-Q+A P)$. Please see [11] for the optimal condition in this case.

\section{Applications}

In the last section, we show how our theorems imply all known general graphic Bernstein type results in higher codimension. In Theorem 1 of [7, the authors proved a Bernstein type theorem in terms of a bound on

$$
\Delta_{f}=\left\{\operatorname{det}\left(\delta_{\alpha \beta}+\sum_{i} f_{x_{\alpha}}^{i}(x) f_{x_{\beta}}^{i}(x)\right)\right\}^{\frac{1}{2}}<2 .
$$

This result improves those in [3] and [5]. This quantity of course is our $\frac{1}{* \Omega}=$ $\sqrt{\prod_{i=1}^{n}\left(1+\lambda_{i}^{2}\right)}$.

It is not hard to check that $\prod_{i=1}^{n}\left(1+\lambda_{i}^{2}\right)<4$ implies $\left|\lambda_{i} \lambda_{j}\right|<1$ for $i \neq j$. Therefore, Theorem A implies the result in [7].

As was mentioned above, Theorem A only assumes that $\Sigma$ is of parallel mean curvature vector. The condition is weakened if we further assume that $\Sigma$ is minimal, i.e., $\sum_{k} h_{\alpha k k}=0$. We demonstrate how it works in two dimensions. Write out the right-hand side of equation (2.5) when $n=2$ :

$$
\begin{aligned}
& |A|^{2}+\lambda_{1}^{2}\left(h_{n+1,1,1}^{2}+h_{n+1,1,2}^{2}\right)+\lambda_{2}^{2}\left(h_{n+2,2,1}^{2}+h_{n+2,2,2}^{2}\right) \\
& +2 \lambda_{1} \lambda_{2}\left(h_{n+1,2,1} h_{n+2,1,1}+h_{n+1,2,2} h_{n+2,1,2}\right) .
\end{aligned}
$$

Since $\Sigma$ is minimal, $h_{n+1,1,1}=-h_{n+1,2,2}$ and $h_{n+2,2,2}=-h_{n+2,1,1}$. This can be shown by completing the square and becomes

$$
|A|^{2}+\left(\lambda_{1} h_{n+1,2,2}+\lambda_{2} h_{n+2,1,2}\right)^{2}+\left(\lambda_{1} h_{n+1,1,2}+\lambda_{2} h_{n+2,1,1}\right)^{2} .
$$

This, in fact, applies to the three-dimensional minimal cone because the second fundamental form vanishes in one direction and the right-hand side of equation (2.5) reduces to the two-dimensional case. This recovers the result of Barbosa [1] and Fischer-Colbrie 3 .

\section{REFERENCES}

[1] Barbosa, Jo ao Lucas Marquês, An extrinsic rigidity theorem for minimal immersions from $S^{2}$ into $S^{n}$, J. Differential Geom. 14 (1979), no. 3, 355-368 (1980). MR 82b:53077

[2] K. Ecker and G. Huisken, A Bernstein result for minimal graphs of controlled growth, J. Differential Geom. 31 (1990), no. 2, 397-400. MR 91a:53010

[3] D. Fischer-Colbrie, Some rigidity theorems for minimal submanifolds of the sphere, Acta Math. 145 (1980), no. 1-2, 29-46. MR 82b:53078

[4] L. Fu, An analogue of Bernstein's theorem, Houston J. Math. 24 (1998), 415-419. MR 2000c:53006

[5] S. Hildebrandt, J. Jost and K.-O. Widman, Harmonic mappings and minimal submanifolds, Invent. Math. 62 (1980/81), no. 2, 269-298. MR 82d:58025

[6] R. Harvey and H. B. Lawson, Calibrated geometries, Acta Math. 148 (1982), 47-157. MR 85i:53058

[7] J. Jost and Y. L. Xin, Bernstein type theorems for higher codimension, Calc. Var. Partial Differential Equations 9 (1999), no. 4, 277-296. MR 2001e:53010

[8] J. Jost and Y. L. Xin, A Bernstein theorem for special Lagrangian graphs, preprint, 2001.

[9] H. B. Lawson, Jr. and R. Osserman, Non-existence, non-uniqueness and irregularity of solutions to the minimal surface system, Acta Math. 139 (1977), no. 1-2, 1-17. MR 80b:35059

[10] Ni, Lei. A Bernstein type theorem for minimal volume preserving maps, Proc. Amer. Math. Soc. 130 (2002), 1207-1210.

[11] M.-P. Tsui and M.-T. Wang, A Bernstein type result for special Lagrangian submanifolds, preprint, 2001. 
[12] M.-T. Wang, Mean curvature flow of surfaces in Einstein four-manifolds, J. Differential Geom. 57 (2001), 301-338.

[13] M.-T. Wang, Long-time existence and convergence of graphic mean curvature flow in arbitrary codimension, Invent. Math. 148 (2002) 3, 525-543.

[14] Y. Yuan, A Bernstein problem for special Lagrangian equation, preprint, 2001.

Department of Mathematics, Columbia University, New York, New York 10027

E-mail address: mtwang@math.columbia.edu 